Military Technical College Kobry El-Kobbah, Cairo, Egypt

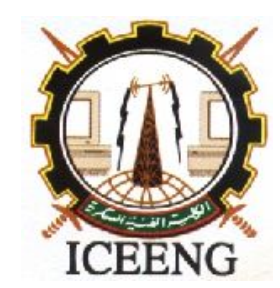

$9^{\text {th }}$ International Conference on Electrical Engineering ICEENG 2014

\title{
Intelligent Laser Angle Detection Using Fuzzy Data Fusion
}

\author{
By
}

\begin{abstract}
Ahmed H.Shemais ${ }^{1}$, Mohamed Elkhatib Yehia Z.Elhalwagy
\end{abstract}

\section{$\underline{\text { Abstract: }}$}

Laser warning receiver is the basic materiel for implementing laser countermeasure. It is very significant with regard to effectively self-protect and destroy the enemy in battlefield. Its function is to detect incidence laser signal, to measure laser parameters. However, Most of the existing system has a complex hardware especially if it gives the direction of the incident laser. In addition, it may face the problem of background sun light suppression. In this paper a simple intelligent algorithm for laser angle detection is proposed using fuzzy logic data fusion to detect laser incidence angle for early warning. The proposed system is simulated and tested using MATLAB software using four laser sensors to detect the incident laser angle from 0 to 360 and an additional sensor to distinguish the incidence laser from background sun light.

\section{Keywords:}

Fuzzy logic systems, laser angle detection, fuzzy data fusion

1- Department of Electrical Engineering, Military Technical College, Egypt,Email: shemais2000@ hotmail.com

2- Department of Electrical Engineering, Military Technical College, Egypt,Email: Mohamed.m.elkhatib@ieee.org

3- Department of Electrical Engineering, Military Technical College, Egypt, Email:johnzake2012@gmail.com 


\section{Introduction:}

One of the major challenges facing military is to accurately identify and locate threats within a secured area. The laser weapons were put into use more widely in the modern battlefield, but the laser menace source was different. The first important mission of the laser countermeasure is to give an alarm of the enemy laser weapons rapidly and rightly[1]. During identifying the laser menaces, realizing the detection of the orientation, the direction of the laser signal and the angle of incidence of laser to determine the source of laser. Then the detect data were fused in virtue of the expert decision making system, and distinguishing of the laser from The background noise of the sun is very serious, to get the aim of correct countermeasure. However, the use of intelligent methods may help solving this problem.

Fuzzy logic methods are capable of fusing uncertain data from multiple sensors to improve the quality of information. They require less computational power than conventional mathematical computational methods such as addition, subtraction, multiplication and division. In addition, only few data samples are required in order to extract final accurate result. Finally, they can be effectively manipulated since they use human language to describe problems[2, 3].

Fuzzy sets and systems have gone through substantial development since the introduction of fuzzy set theory by Zadeh [4-8] about four decades ago. They have found a great variety of applications ranging from control engineering, qualitative modeling, pattern recognition, signal processing, information Processing, machine intelligence, decision making, management, finance, medicine, motor industry, robotics, and so on [9-15] .

In this paper, a data fusion algorithm based on fuzzy logic methods to detect angle of incidence of laser and enhance the performance of the sensors is proposed.

The rest of the paper is organized as follows. In Section 2, we present an overview of fuzzy logic systems and data fusion, the proposed algorithm are described in Section 3, Simulation setup and Performance are described in section 4 , Lastly, we conclude in Section 5.

\section{2.fuzzyOverview of Fuzzy Data Fusion System}

\subsection{Introduction TO FUZZY LOGIC System}

The main motivation for fuzzy logic is that by simply writing down common sense rules it is possible to build a reasonable control strategy without deep theoretical knowledge of control [16]. The basic fuzzy logic system is composed of a set of input membership functions, a rule-based controller, and a defuzzification process. The fuzzy logic input uses member functions to determine the fuzzy value of the input. There can be any 
number of inputs to a fuzzy system and each one of these inputs can have several membership functions. The set of membership functions for each input can be manipulated to add weight to different inputs. The output also has a set of membership functions. These membership functions define the possible responses and outputs of the system [17].

Fig. 1 shows the block diagram of a fuzzy control system.

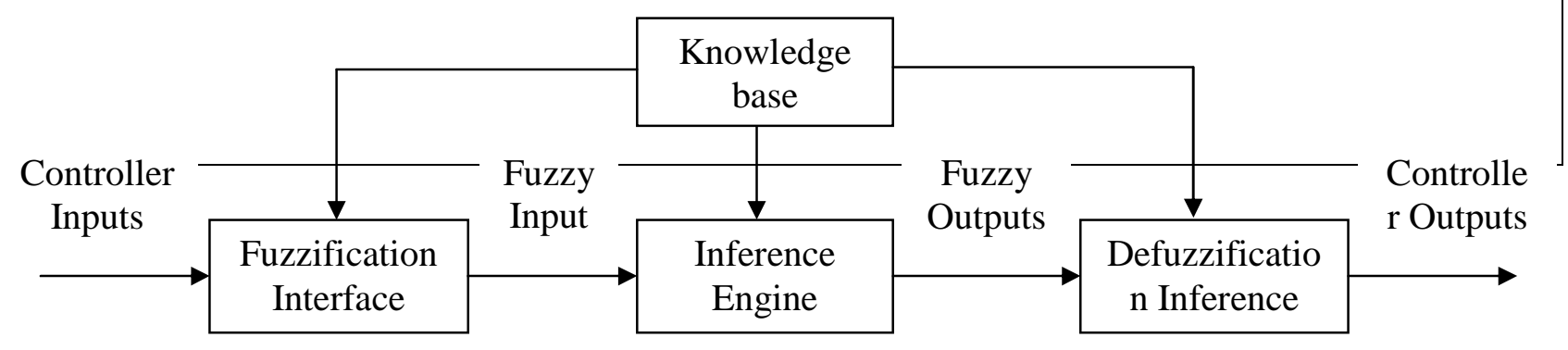

Figure (1). Basic structure of fuzzy

The fuzzy inference engine is the heart of the fuzzy logic control system. It is a rule based controller that uses If-Then statements to relate the input to the desired output [4]. The fuzzy inputs are combined based on these rules and the degree of membership in each function set. The output membership functions are then manipulated based on the controller for each rule. Several different rules will usually be used since the inputs will usually be in more than one membership function. All of the output member functions are then combined into one aggregate topology. The defuzzifaction process then chooses the desired finite output from this aggregate fuzzy set. There are several ways to do this such as weighted averages, centroids, or bisectors. This produces the desired result for the output.

There are countless different membership function topologies that can be used, the most common are triangular, Gaussian, trapezoidal, S-shaped and Z-shaped. [18]

The laser alarm device identified the enemy laser menace source by fuzzy decision making system. This system was composed by the Fuzziness, the Making Fuzzy Decision and De-fuzziness, as for Fig 2. The Fuzziness was conversing the precise quantity to fuzzy quantity, the De-fuzziness was conversing the fuzzy quantity to precise quantity, and the Making Fuzzy Decision was the fuzzy relation between the input and output. The three parts were realized by soft programming.[19] 


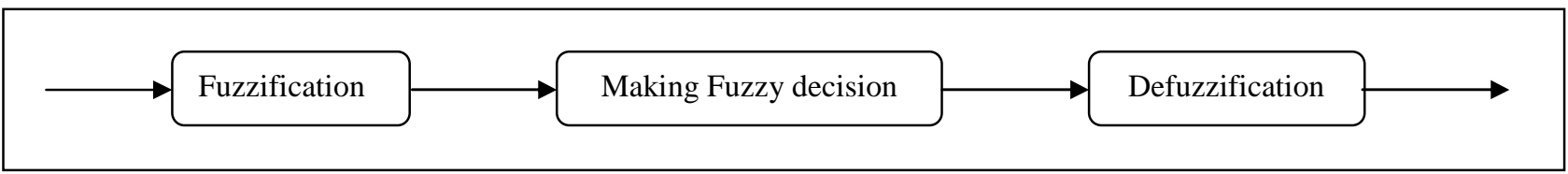

Figure (2). Diagram of fuzzy decision system

A fuzzy set is a set where degrees of membership between 1 and 0 are allowed; it allows partial membership. Fuzzy sets can thus better reflect the way intelligent people think. For example, an intelligent person will not classify people as either friends or enemies; there is a range between these two extremes. Not recognizing that there are degrees in every trait can lead to erroneous decisions. Vague human expressions such as tall, hot, cold, etc. can be expressed by fuzzy sets [18].

The choosing of rule of fuzzy was the key part in the designing fuzzy controller. It included choosing proper fuzzy variable, conforming the degree of membership function of each fuzzy variable, and establishing the rule of fuzzy control. The fuzzy rules library was composed by many fuzzy rules. It can be expressed by IF-THEN. For example, IF $<$ FPI $>$ THEN $<$ FP2 $>$ can express a fuzzy rule, FPI and FP2 were fuzzy proposition.[19]

\subsection{Sensors Fuzzy DATA FUSION}

Data fusion is the process of integration of multiple data and knowledge representing the same real-world object into a consistent, accurate, and useful representation.

Fusion of the data from two sources (dimension \#1 \& \#2) can yield a classifier superior to any classifiers based on dimension \#1 or dimension \#2 alone as shown in Fig.3

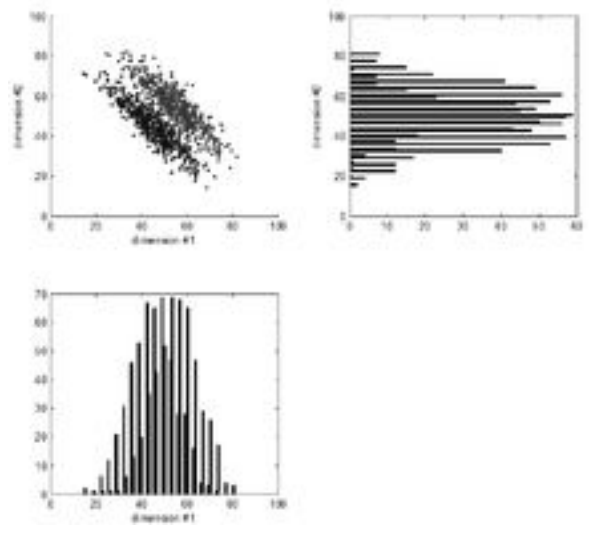

Figure.(3) Data fusion from two sources 
Data fusion processes are often categorized as low, intermediate or high, depending on the processing stage at which fusion takes place. Low level data fusion combines several sources of raw data to produce new raw data. The expectation is that fused data is more informative and synthetic than the original inputs.[2, 3]

For example, sensor fusion is also known as (multi-sensor) data fusion and is a subset of information fusion.

In the framework of fuzzy set and possibility theory the modeling step consists in defining a membership function to each class or hypothesis in each source, or a possibility distribution over the set of hypotheses in each source. Such models explicitly represent imprecision in the information, as well as possible ambiguity between classes or decisions.

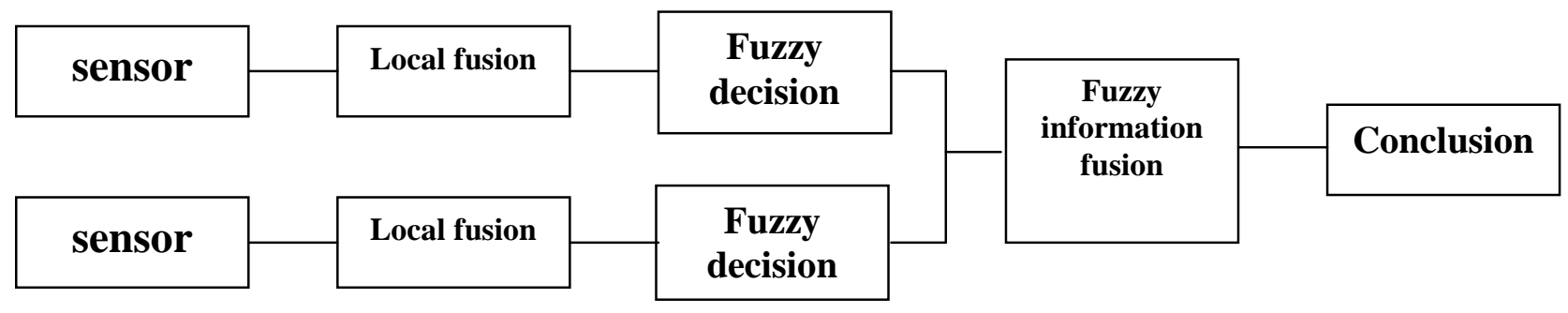

Figure (4). Structure of the information

Therefore, as shown in Figure 4 Fuzzy Data fusion techniques combine data from multiple sensors, and related information from associated databases, to achieve improved accuracies and more specific inferences than could be achieved by the use of a single sensor alone $[20,21]$

\section{The Proposed Laser Angle Detection Fuzzy Model}

The proposed algorithm is based on the fuzzy logic inference methods described in previous section. The fuzzy model for the laser angle detection diagnostic was implemented in MATLAB using the fuzzy logic toolbox. The proposed Simulink model has five different inputs: the Right, Left, Upper, Down and Dummy inputs which come from the sensors organized in the basic directions as shown in Fig.5. These five inputs are processed by a fuzzy logic algorithm that gives the angle of incidence of laser as an output.

This resulting angle is decoded into one of nine possible outputs: UU(upper), UR (between upper and right angles with fuzzy values), RR(right), RD (between right and down), DD(down), LD (between left and down), LL(left), UL (between left and down) and NONE (no dangerous).

Using fuzzy rules and output membership functions the exact output angle is produced by fuzzy data fusion. 


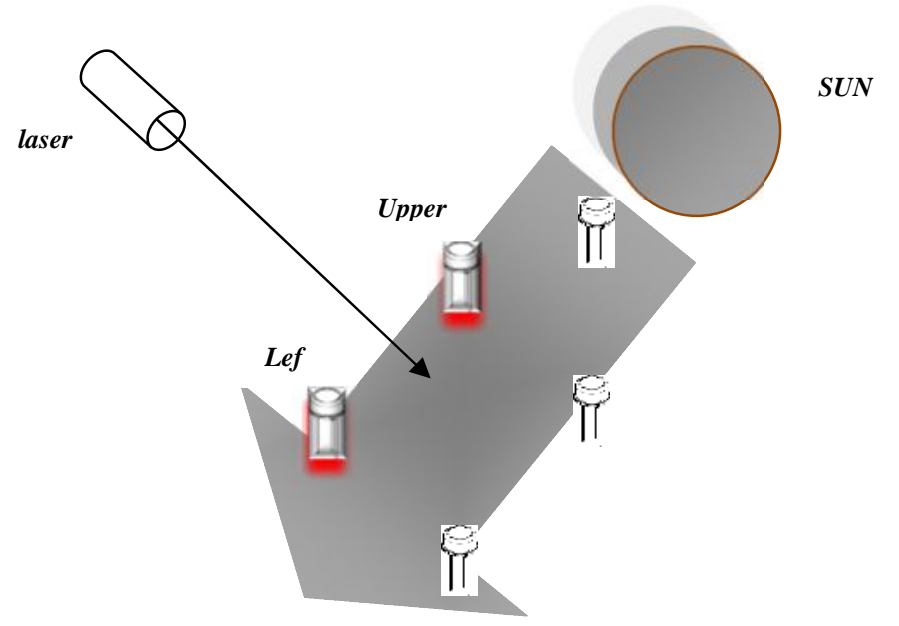

Figure 5. The proposed system sensors configuration.

\subsection{The proposed system block diagram}

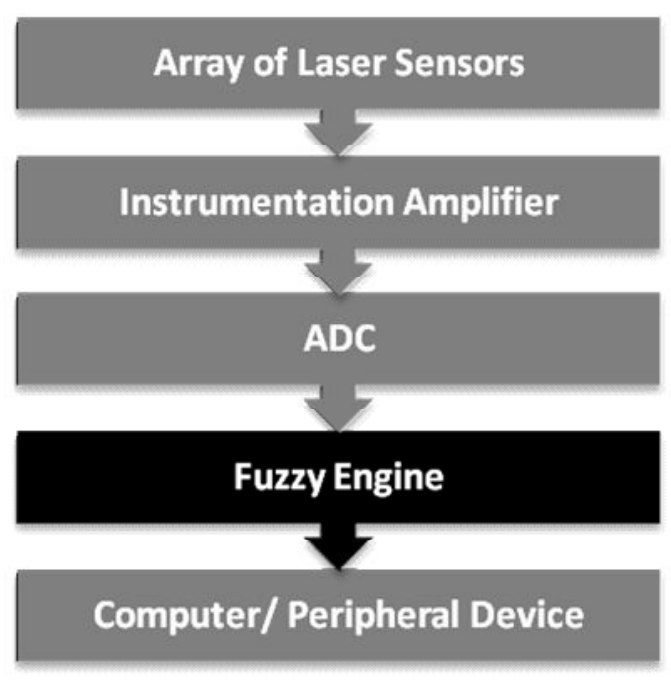

Figure 6. The proposed system block diagram

This diagnostic system uses Signal builder inputs (which allow to create and generate interchangeable groups of signals whose waveforms are piecewise linear) for the five inputs (upper, right, down, left and dummy). Each input has a certain signal in the range from 0 to 255 represents the strength of the incident laser. The signal from the sensors then go the interface circuit that condition the signal to be processed by the microcontroller which as the fuzzy algorithm. Finally the output is displayed. (refer to block diagram shown in Fig.6) 


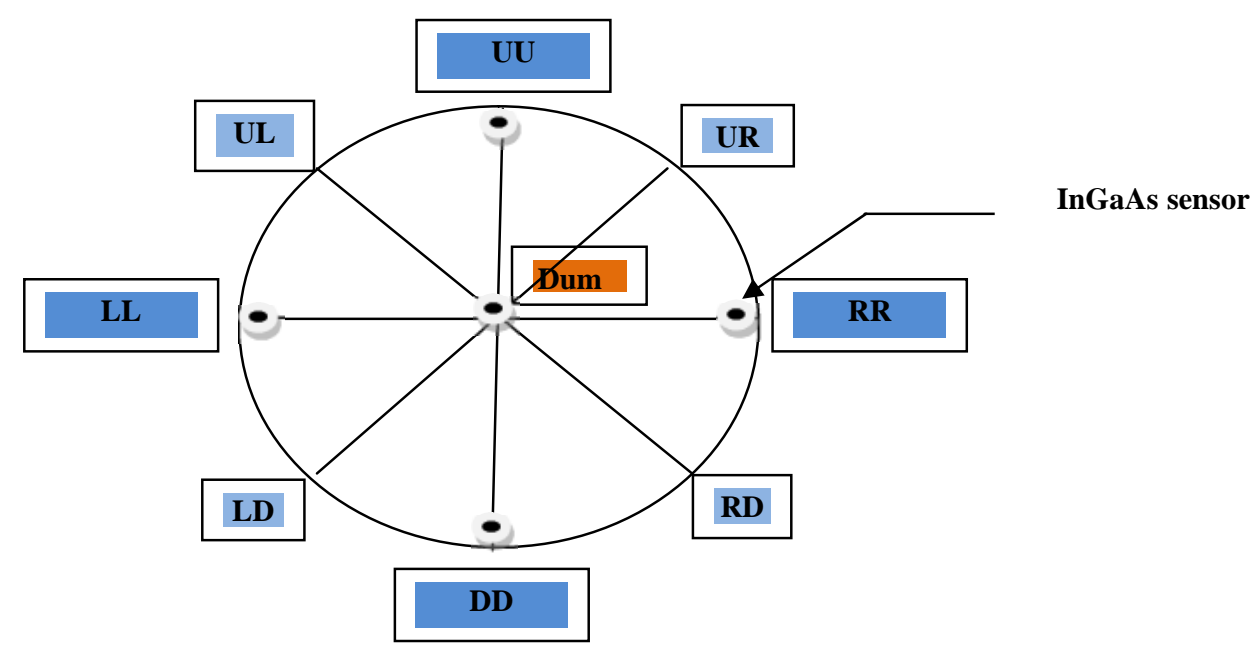

Figure.7 Sensors configuration and orientations

Figure7 shows sensors configuration used to cover all domain angles from 0 to 360 degree. Each sensor indicates a direction as follow:

Upper Sensor (UU) is at 0 or 360 degree, Right sensor (RR) is put at 90 degree, Down sensor (DD) is set at 180 degree and Left senor (LL) is at 270 degree. In order to increase the resolution and make fuzzy data fusion the following directions have been defined:

UR is the angle between upper and right sensors; UL is the angle between upper and left sensors; RD is the angle between right and down sensors and LD is the angle between left and down sensors.

For the fuzzy data fusion algorithm, and in order to get the right angle depending on the sensors readings, some comparisons between the sensors readings have been set as follow:

1- Error between Upper and right readings

2- Error between Upper and left readings

3- Error between Down and right readings

4- Error between Down and left readings

5- The dummy sensor illuminates the sun effect by exposing it directly to the sun ...so we can indicate the incidence of the laser by comparing all the sensors with the dummy sensor. 


\subsection{Membership functions for the fuzzy model of laser angle detection}

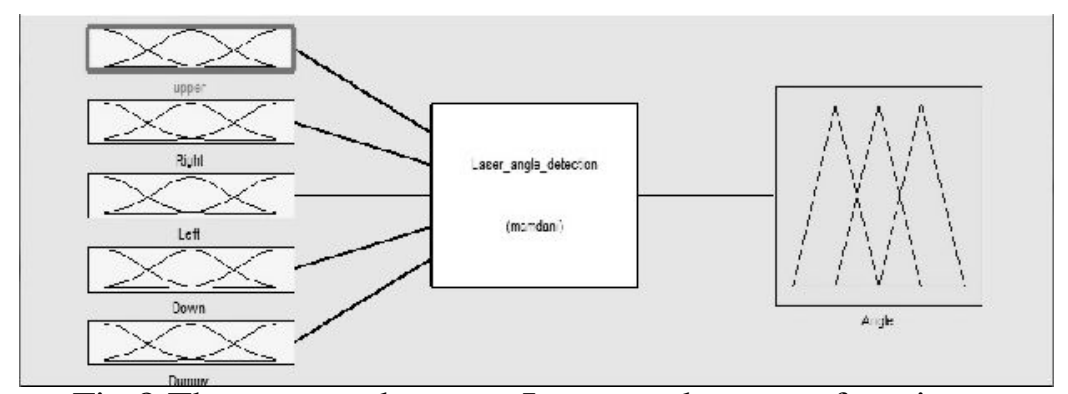

Fig.8 The proposed system Inputs and outputs functions

These inputs function ranges (sensors readings ranges) can be used in determining the fuzzy membership sets. The fuzzy system will have five inputs and one indicating output (Figure 8). The fuzzy system uses Mamdani type fuzzy system [4][22, 23], and the centroid method for defuzzification. The input membership functions for the four sensors (upper, down, left, right) are shown in Fig. 9. Each sensor input has three different membership functions: low, medium and high. They ranges from 0 to 255, which are the possible input values, came from the real laser detector. The low and high membership functions continue to infinity to include any voltage value out of range and avoid the system saturation.
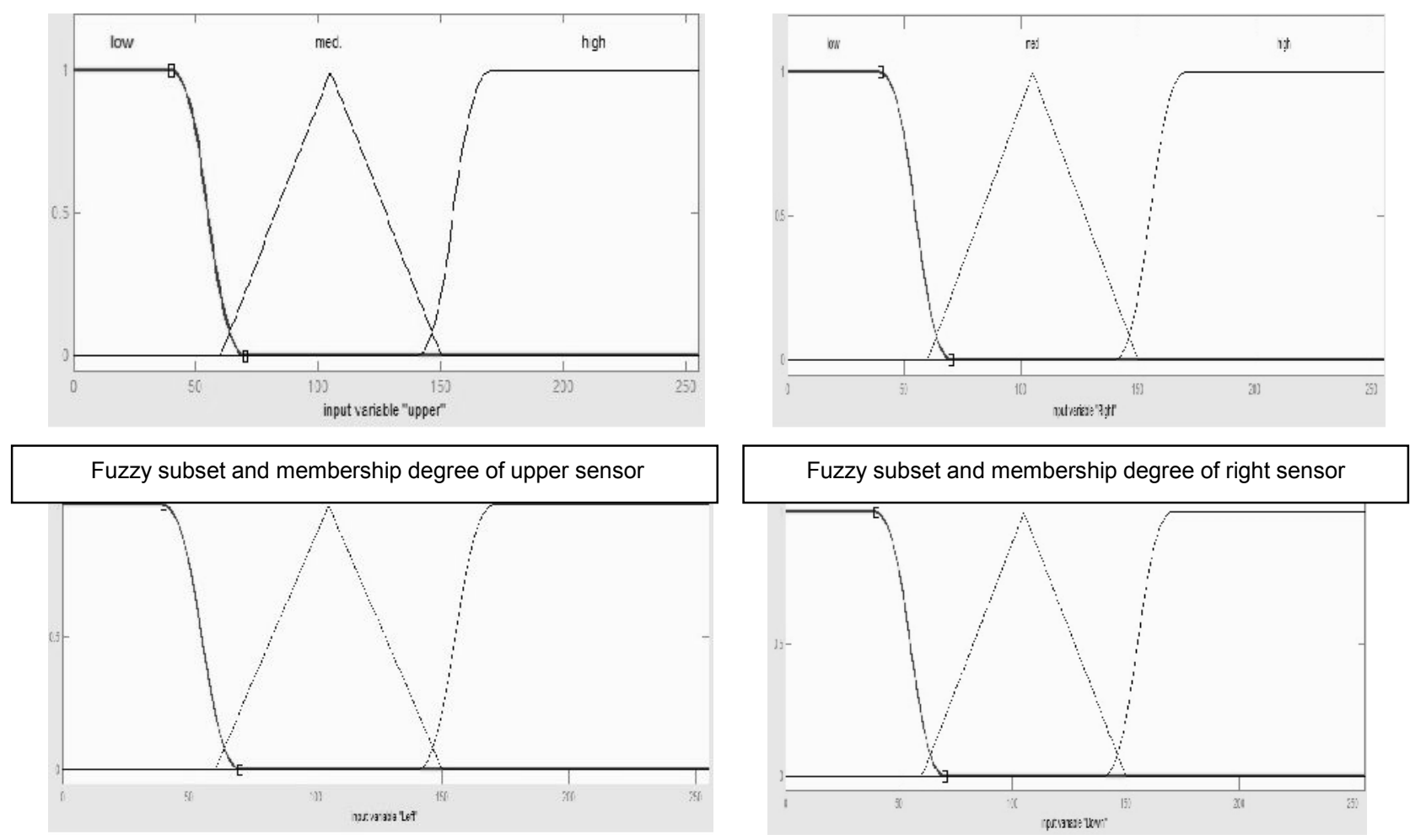
The membership de! Figure. 9 The Membership function for the input he four sensor inputs can be expres:

Low: $\boldsymbol{\mu u u}_{\mathrm{uu}}(\mathrm{x}: 40,70)$

Med.: $\boldsymbol{\mu u u}(\mathrm{x}: 60,105,150)$

High: $\boldsymbol{\mu u u}(\mathrm{x}: 140,170)$

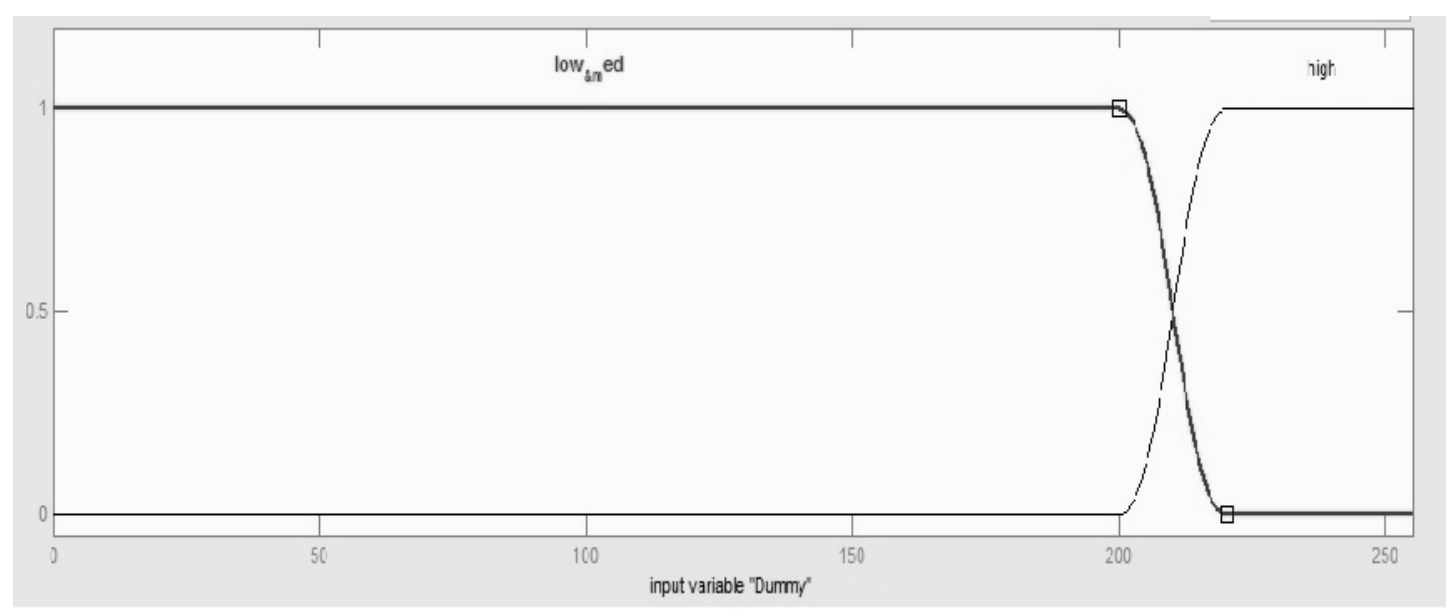

The dummy sensor input readings can be divided into two fuzzy sets as shown in Fig.10. They are defined as low_medium function (using Z-shaped membership degree) ranges from 0 to 220 and high function (using S-shaped membership degree) ranges from 200 to 255 as high. The membership degree of two fuzzy sets can be expressed as followed:

Low_med.: $\boldsymbol{\mu}$ dummy $(x: 000,220)$

High: $\boldsymbol{\mu}_{\text {dummy }}(\mathrm{x}: 220,255)$ 


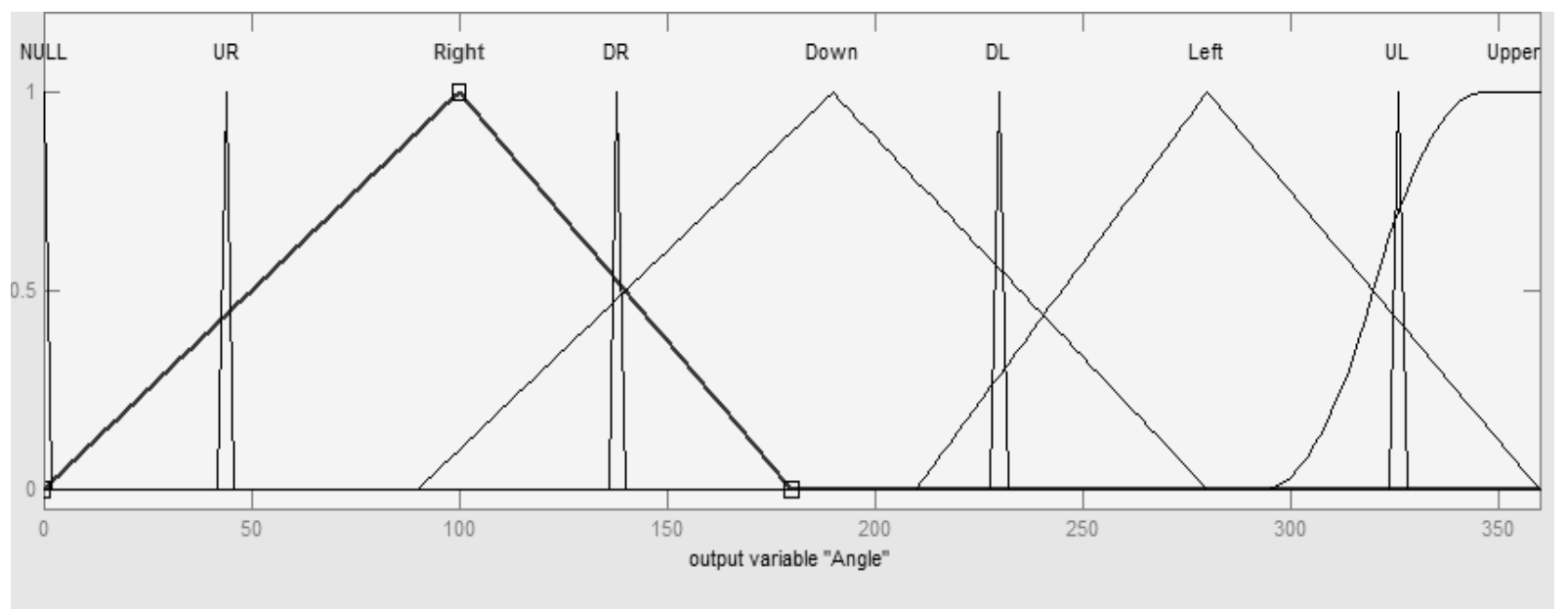

$\mathrm{Th}$

Figure 11. Fuzzy subset and membership degree of the output angle

and right), RR (right), RD (angle between right and down), DD (down), LD (between left and down), LL (left), UL (between left and upper again) and NONE (no dangerous). The output membership functions are triangular shape and cover the range from 0 to 360 as shown in Fig.11.

Once all of the input and output membership functions have been defined the heart of the control can now be defined; the rules. The fuzzy rules are in the form of if-then statements.

These statements look at both inputs and determine the desired output. In this system when laser beam incidence occur on one of the sensors so the output reading of this sensor will be compared with the output of the dummy sensor (exposed directly to the sun) to distinguishing the laser from The background noise of the sun, to get the aim of correct countermeasure and in the same time compared also with the other neighbor sensor. As one of the advantages of the fuzzy system is the parallel processing of the data at the same time.

Examples of the rules defined for this system are in Table 1 below:

Table 1 Examples of the fuzzy rule for the proposed system.

\begin{tabular}{|l|l|l|l|}
\hline If Upper & And Right & And Dummy & Then The Output Angle \\
\hline Low & Low & Low & None \\
\hline Medium & Low & Low & Upper \\
\hline Medium & Medium & Low & UR \\
\hline High & Low & Low & upper \\
\hline
\end{tabular}


The fuzzy rules have been set such that it makes use of all the data and ensure data fusion of all the sensor readings. The rules were formulated one-by-one, and then the whole rules-set was analyzed to make it:-

- Complete: any combination of the inputs fired at least one rule.

- Consistent: contains no contradictions.

- Continuous: has no neighboring rules with output fuzzy sets that have an empty intersection

\section{System Simulation and Testing}

The proposed system has been designed and simulated on Simulink software using different combinations of input readings of the sensors to ensure the prober working of the system. The next table (table 2) shows examples of the simulation results; input and output combinations for the basic direction sensors. While Table 3 shows another combinations of sensors readings to test the in between directions data fusion of the proposed system.

Table (2) Example results of simulation for the basic direction sensors

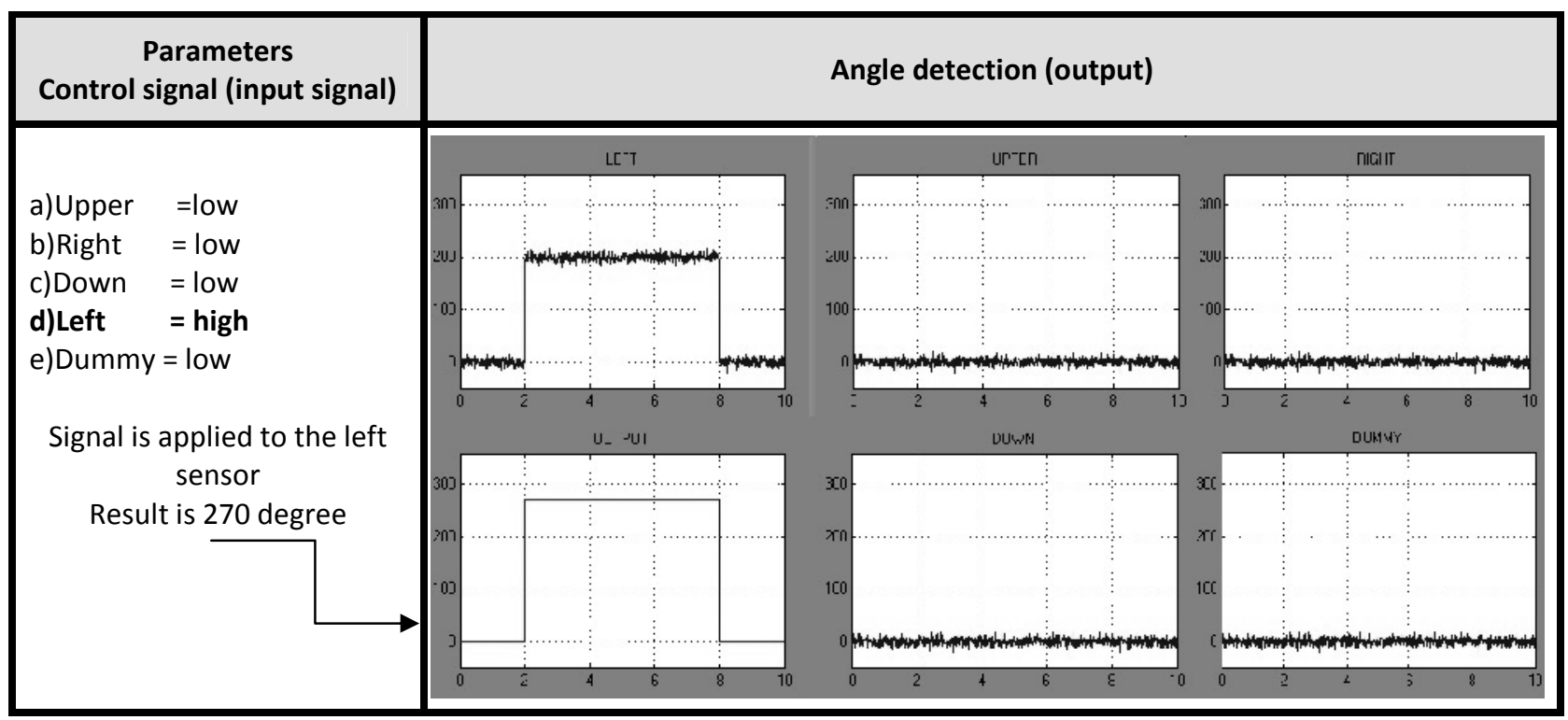


a ) Upper =high

b) Right = low

c) Down =low

d ) Left = low

e)Dummy = low

Signal is applied to the upper sensor Result is 320 degree

a) Upper =low

b) Right = high

c) Down = low

d ) Left = low

e) Dummy = low

Signal is applied to the right sensor Result is 90 degree
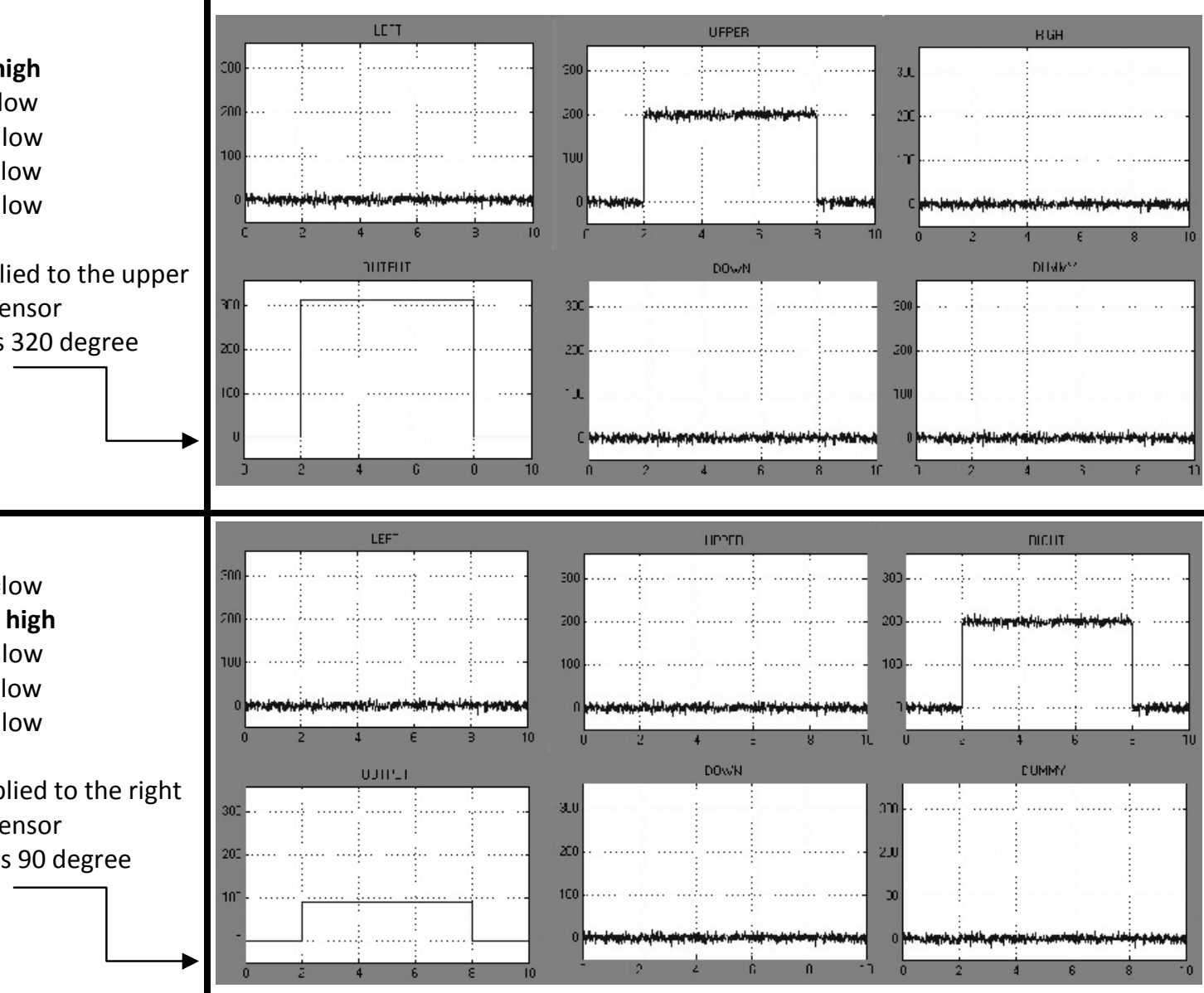

Table (3) Example results of simulation of in between direction of the basic directions

\begin{tabular}{|c|c|}
\hline Parameters & Angle detection (output) \\
\hline Control signal (input signal) & \\
\hline
\end{tabular}



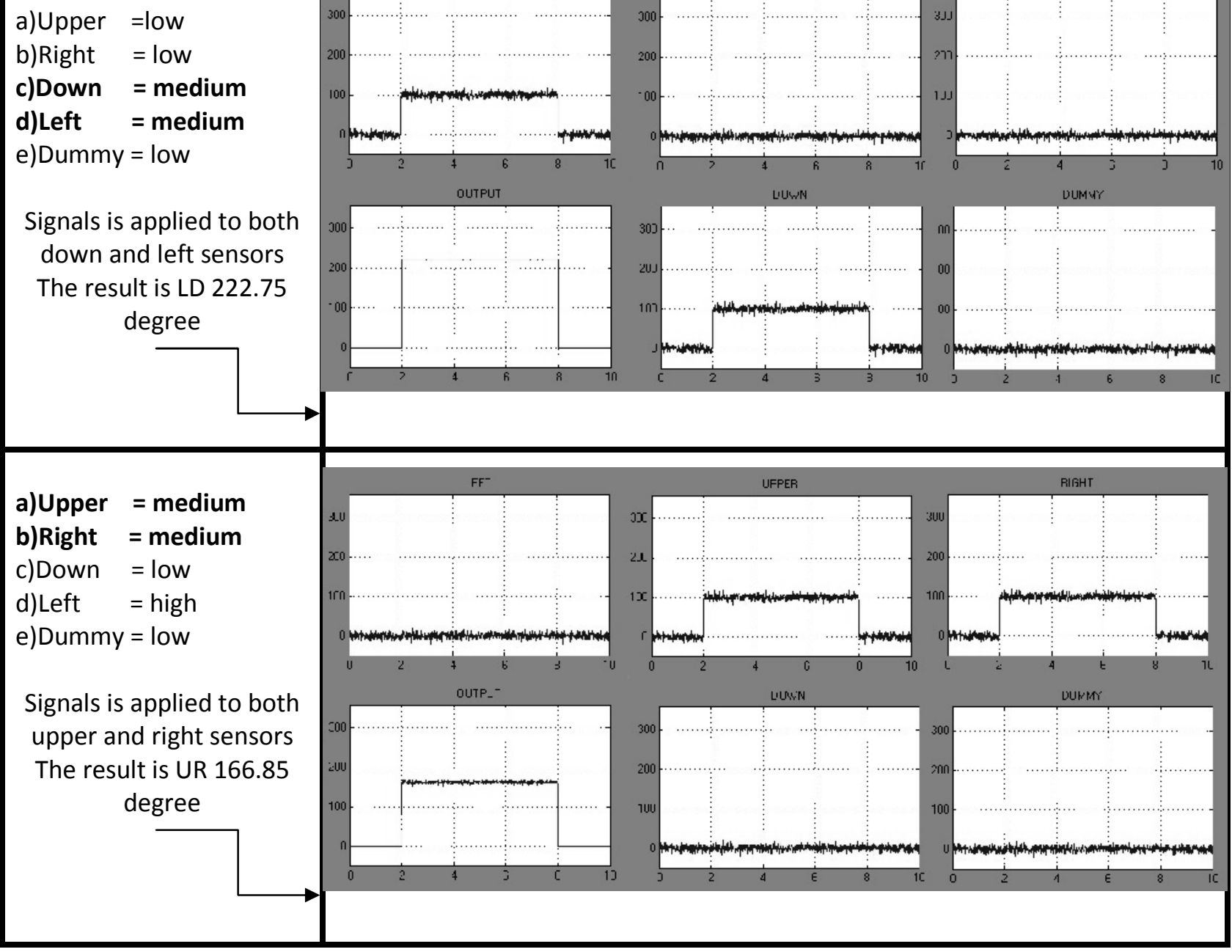

\section{Conclusions:}

The fuzziness mathematical models of laser angle detection parameters and fuzzy decision rules library of identifying laser angle are built, which are set up by using fuzzy decision theory. Professional system of fuzzy decision is designed using multiple 
sensors orientation.

The objective of this work was building model of fuzzy logic controller for laser detection using multiple sensors. The proposed model of laser angle detection system was built in Matlab - Simulink. Simulation software and the proposed fuzzy logic system was designed using the Fuzzy Logic Toolbox.

The simulation results are tested and show good results.

This research addresses a problem of how to make effective use of real-time information acquired from multiple sensors in military battlefield .

\section{References}

1. Li, X., et al. A new design for laser warning system. in Proceedings of the 7th WSEAS International Conference on Signal, Speech and Image Processing. 2007. World Scientific and Engineering Academy and Society (WSEAS).

2. Khaleghi, B., et al., Multisensor data fusion: A review of the state-of-the-art. Information Fusion, 2013. 14(1): p. 28-44.

3. Klein, L.A., Sensor and data fusion: a tool for information assessment and decision making. Vol. 324. 2004: SPIE press Bellingham^ ${ }^{\wedge}$ WA WA.

4. Zadeh, L.A., Fuzzy sets. Information and control, 1965. 8(3): p. 338-353.

5. Zadeh, L.A., The concept of a linguistic variable and its application to approximate reasoning - I. Information sciences, 1975. 8(3): p. 199-249.

6. Zadeh, L.A., Similarity relations and fuzzy orderings. Information sciences, 1971. 3(2): p. 177-200.

7. Zadeh, L.A., Outline of a new approach to the analysis of complex systems and decision processes. Systems, Man and Cybernetics, IEEE Transactions on, 1973(1): p. 28-44.

8. Zadeh, L.A., Fuzzy algorithms. Information and control, 1968. 12(2): p. 94-102.

9. LEE, C., Fuzzy logic in control systems: fuzzy logic controller-part I. IEEE Trans. Syst., Man, Cybern., 1990. 20(2): p. 404-418.

10. Lee, C.-C., Fuzzy logic in control systems: fuzzy logic controller. II. Systems, Man and Cybernetics, IEEE Transactions on, 1990. 20(2): p. 419-435.

11. Kosko, B., Neural networks and fuzzy systems1992: Prentice hall.

12. Dutta, S., Fuzzy logic applications: Technological and strategic issues. Engineering Management, IEEE Transactions on, 1993. 40(3): p. 237-254.

13. Bonissone, P.P., et al., Industrial applications of fuzzy logic at General Electric. Proceedings of the IEEE, 1995. 83(3): p. 450-465.

14. Babuška, R. and H. Verbruggen, An overview of fuzzy modeling for control. Control Engineering Practice, 1996. 4(11): p. 1593-1606.

15. Keller, J., R. Krisnapuram, and N.R. Pal, Fuzzy models and algorithms for 
pattern recognition and image processing. Vol. 4. 2005: Springer.

16. Klingenberg, B.R. and P.F. Ribeiro. Fuzzy Logic for Harmonic Distortion Diagnosis in Power Systems. in Electro/information Technology, 2006 IEEE International Conference on. 2006. IEEE.

17. Nuguyen, T., C. Walker, and E. Walker, A first course in fuzzy and neural control. Boca Raton, FL, 2003.

18. Ibrahim, A., Fuzzy logic for embedded systems applications2003: Newnes.

19. Tao, S. and S. Jian-she. Laser menace identifying based on fuzzy decision. in Fuzzy Systems and Knowledge Discovery, 2007. FSKD 2007. Fourth International Conference on. 2007. IEEE.

20. Klein, L.A. Sensor and data fusion concepts and applications. 1993. Society of Photo-Optical Instrumentation Engineers (SPIE).

21. Klein, L.A. Sensor and data fusion concepts and applications. 1999. Society of Photo-Optical Instrumentation Engineers (SPIE).

22. Mamdani, E.H. Application of fuzzy algorithms for control of simple dynamic plant. in Proceedings of the Institution of Electrical Engineers. 1974. IET.

23. Mamdani, E.H. and S. Assilian, An experiment in linguistic synthesis with a fuzzy logic controller. International journal of man-machine studies, 1975. 7(1): p. 113. 